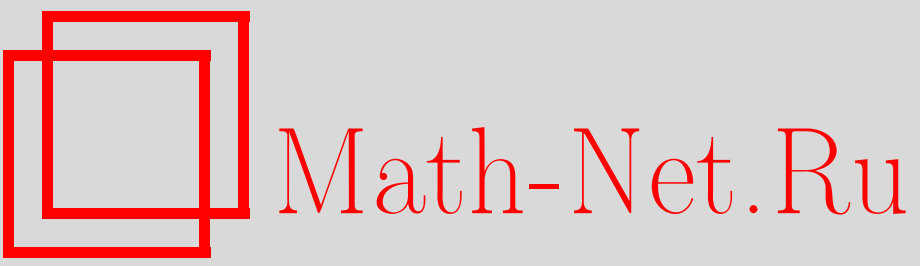

Г. И. Калмыков, Представление вириальных коэффициентов, позволяющее избежать асимптотической катастрофы, ТМФ, 2002, том 130, номер 3, 508-528

DOI: https://doi.org/10.4213/tmf316

Использование Общероссийского математического портала Math-Net.Ru подразумевает, что вы прочитали и согласны с пользовательским соглашением

http://www.mathnet.ru/rus/agreement

Параметры загрузки:

IP: 54.162 .85 .209

26 апреля 2023 г., 17:29:48 


\section{ПРЕДСТАВЛЕНИЕ ВИРИАЛЬНЫХ \\ КОЭФФИЦИЕНТОВ, ПОЗВОЛЯЮЩЕЕ ИЗБЕЖАТЬ АСИМПТОТИЧЕСКОЙ КАТАСТРОФЫ}

Рассмотрено понятие асимптотической катастрофы в представлениях майеровских коэффициентов. Указаны проявления этой катастрофы и дано ее формальное определение. Объяснена значимость введенного определения асимптотической катастрофы. Даны представления вириальных коэффициентов, в которых отсутствует феномен асимптотической катастрофы. Представления основаны на введенном разложении множеств помеченных неразделимых в смысле Харари графов (блоков) на классы, маркируемые ансамблями циклов, удовлетворяющими введенным условиям и названными ансамблями каркасных циклов. Эти же классы могут маркироваться специальными блоками, названными каркасами. Каркасы ставятся во взаимно однозначное соответствие указанным ансамблям циклов и играют в каркасной классификации блоков роль, аналогичную роли деревьев в древесной классификации связных помеченных графов. Введена древесная классификация ансамблей каркасных циклов. Доказано, что данные представления вириальных коэффициентов свободны от явления асимптотической катастрофы.

\section{1. ВВЕДЕНИЕ}

В статье рассматриваются термодинамически равновесные системы классических частиц, взаимодействующих центральными парными силами в $\nu$-мерном действительном евклидовом пространстве $\mathbb{R}^{\nu}$. Известно [1], [2], что уравнение состояния таких систем представляется в параметрическом виде (разложения давления $p$ и плотности $\rho$ в ряды по степеням активности $z$ ). Коэффициенты этих разложений при $z^{n}$ обычно выражаются (см. [1], [2]) суммами интегралов, маркируемых помеченными связными графами [3] (см. также приложение A), причем суммирование производится по всем помеченным $n$-вершинным связным графам. Такое представление коэффициентов разложений по активности мы будем называть традиционным представлением.

В работах [4]-[6] показано, что традиционное представление коэффициентов разложений давления и плотности по степеням активности имеет сушественный недостаток: оно обладает свойством, которое в [4], [5] названо асимптотической катастрофой.

* Центр физических исследований им. П. Н. Лебедева, Москва, Россия 
Суть асимптотической катастрофы состоит в следующем. С одной стороны, число слагаемых в сумме, представляющей $n$-й коэффициент, равно числу всех связных $n$-вершинных помеченных графов и при $n \rightarrow \infty$ растет катастрофически быстро, как величина $2^{n(n-1) / 2}$. С другой стороны, сам $n$-й коэффициент по модулю не может превосходить гораздо более медленно растушую величину $A^{n}$ (где $A>1$ ) вследствие хорошо известного факта сходимости разложений давления и плотности по степеням активности в некотором круге ненулевого радиуса с центром в начале координат [2], [7]. Этот коэффициент представляется суммой, в которой модули всех слагаемых ограничены снизу величиной, хотя и убываюшей с ростом $n$, но не катастрофически быстро, т.е. медленнее, чем любая из величин вида $B^{n} 2^{-n(n-1) / 2}$, где $B$ - положительная константа (строгое доказательство этого факта для неотрицательных потенциалов с твердым ядром дано в работе [6]). Следовательно, сходимость упомянутых степенных рядов достигается только за счет того, что в сумме, традиционно представляющей $n$-й коэффициент, подавляющая часть слагаемых с большой точностью взаимно уничтожаются как величины противоположных знаков [5], [6]. После такого взаимного уничтожения остается сравнительно небольшой остаток, являюшийся при $n \rightarrow \infty$ бесконечно малой величиной по сравнению с числом слагаемых в сумме, традиционно определяющей этот коэффициент. Этот остаток, представляюший основной интерес, даже при небольших $n$ становится недоступным для непосредственного исследования. В частности, произведение числа слагаемых в сумме, традиционно представляющей $n$-й коэффициент, на некоторое характерное слагаемое не дает никакого представления ни о величине $n$-го коэффициента, ни о его асимптотике.

Рассматриваемые коэффициенты, за исключением отдельных частных случаев нереалистических потенциалов, приходится находить путем численного расчета, например методом Монте-Карло. В работе [6] показано, что асимптотическая катастрофа приводит к практически неибежному катастрофически быстрому росту ошибок вычислений этих коэффициентов с возрастанием $n$. В этом причина того, что до настоящего времени никому не удалось более или менее точно вычислить даже шестой (!) коэффициент.

Асимптотическая катастрофа имеет место также в обычных представлениях коэффициентов вириального разложения давления по степеням плотности (вириальных коэффициентов) [1], [8], [9], коэффициентов разложений по степеням активности z усеченных корреляционных функций [2], [10], [11], а также в представлениях коэффициентов обобщенных разложений свободной энергии по степеням плотности $\rho$, в которых коэффициенты при $\rho^{n}$ зависят от $\rho[4],[12]-[27]$.

Первый успех в деле устранения асимптотической катастрофы достигнут в работе [28] (см. также [29]), где асимптотическая катастрофа устранена из представлений коэффициентов разложений давления $p$ и плотности $\rho$ по степеням активности $z$. Образно говоря, в работе [28] удалось путем теоретического анализа в явном виде осуществить упомянутое выше "взаимное уничтожение" избыточных слагаемых в суммах, выражающих коэффициенты этих степенных рядов по активности.

Не вдаваясь пока в детали, поясним эффект устранения асимптотической катастрофы 
ТАБЛИЦА 1

\begin{tabular}{|c|c|c|c|}
\hline$n$ & 4 & 5 & 6 \\
\hline$s_{t}(n)$ & 16 & 125 & 1296 \\
\hline$s_{m}(n)$ & 38 & около 1000 & около 30000 \\
\hline
\end{tabular}

из разложений по активности с помощью табл. 1 , где через $s_{m}(n)$ обозначено число слагаемых в традиционном представлении $n$-го коэффициента разложений по активности, а через $s_{t}(n)$ - число слагаемых в представлении [28]. Табл. 1 наглядно демонстрирует, что представление [28] гораздо экономнее традиционного представления этих коэффициентов.

Представление [28] удобно не только тем, что уменьшается число слагаемых в суммах, выражающих коэффициенты рядов, но еще и тем, что благодаря устранению асимптотической катастрофы эти коэффициенты становятся доступными непосредственному аналитическому исследованию. В частности, в случае неотрицательного потенциала совершенно тривиально получаются оценки снизу и сверху радиуса сходимости рядов, которые ранее в литературе производились путем сложных обходных построений [2], [30] . Так, в работе [28] дается оценка снизу радиуса сходимости, получаемая, если модуль $n$-го коэффициента ряда оценить сверху как произведение числа слагаемых (равного числу $n^{n-2}$ помеченных деревьев $\mathrm{c} n$ вершинами) на максимальное значение модуля слагаемого. Оценка сверху радиуса сходимости рядов в [28] получается, если модуль $n$-го коэффишиента ряда оценить снизу как произведение числа линейных помеченных деревьев на значение модуля слагаемого, помеченного линейным деревом (под линейным понимается дерево, представляюшее собой простую цепь).

Цель настоящей работы - построить схему, логически аналогичную конструкции [28], которая эффективно устраняла бы асимптотическую катастрофу из представлений вириальных коэффициентов.

Полученный результат продемонстрирован в табл. 2 , где $s_{v, m}(n)$ - число слагаемых в традиционном представлении $n$-го вириального коэффициента, $s_{v, f}(n)$ - число слагаемых в представлении этого коэффициента, построенного ниже. Видно, что по крайней мере для значений $n=4,5,6$ представление, строящееся ниже, несравненно экономнее традиционного представления вириальных коэффициентов.

ТАБЛИцА 2

\begin{tabular}{|c|c|c|c|}
\hline$n$ & 4 & 5 & 6 \\
\hline$s_{v, f}(n)$ & 5 & 49 & 784 \\
$s_{v, m}(n)$ & 10 & около 1000 & около 30000 \\
\hline
\end{tabular}

По нашему мнению, новое представление позволит вычислить пятый и шестой вириальные коэффициенты для реалистических потенциалов достаточно точно при приемлемом объеме вычислений. 
Перейдем теперь к объяснению математического аппарата, при помощи которого в работе [28] устранена асимптотическая катастрофа из разложений по активности. Такое пояснение здесь уместно, так как математический аппарат, строящийся в данной статье, логически является непосредственным обобшением аппарата, примененного в работе [28].

Основой построенного в работе [28] представления коэффициентов разложений давления и плотности по активности является древесная классификация связных помеченных графов. Объясним вкратце суть этой классификации. В качестве меток классов, на которые разбивается множество $F(n)$ всех помеченных связных графов с множеством вершин $V_{n}=\{1,2, \ldots, n\}$, выступают помеченные деревья с множеством вершин $V_{n}$. Каждому такому дереву $t$ ставится в соответствие определенное в приложении А множество $X_{\mathrm{ad}}(t)$ допустимых для этого дерева ребер. Каждое ребро из этого множества соединяет две несмежные вершины дерева $t$.

Далее, каждому помеченному дереву $t$ с множеством вершин $V_{n}$ ставится в соответствие множество $F(t)$ связных помеченных графов с множеством вершин $V_{n}$, состояшее из дерева $t$ и всех его надграфов, каждый из которых можно получить добавлением к дереву $t$ одного или нескольких ребер из множества $X_{\mathrm{ad}}(t)$ допустимых для этого дерева ребер. При этом каждый граф̆ из множества $F(n)$ принадлежит одному и только одному из множеств вида $F(t)$. Отсюда следует, что совокупность $F(n)$ всех связных помеченных графов с множеством вершин $V_{n}$ разлагается на непересекаюшиеся множества, т.е. классы, метками которых являются помеченные деревья с множеством вершин $V_{n}$.

Эта классификация была введена ${ }^{1)}$ в [28] и называется древесной классификацией связных помеченных графов.

Используя древесную классификацию, $n$-й коэффициент можно представить в виде интеграла от повторной суммы, где внешнее суммирование производится по всем помеченным деревьям $t$ с множеством вершин $V_{n}$, а внутреннее суммирование - по всем графам из множества $F(t)$. Во внутренней сумме произведение множителей, помеченных ребрами дерева $t$, может быть вынесено как обший множитель. В оставшейся внутренней сумме слагаемые представляют собой произведения множителей, помеченных ребрами из множества $X_{\mathrm{ad}}(t)$, а суммирование производится по всем подмножествам множества $X_{\text {ad }}(t)$, включая и пустое множество. Поэтому внутренняя сумма свертывается в одно произведение всех больцмановских экспонент, определенных на допустимых ребрах дерева $t$. Такое свертывание суммы в произведение является чем-то вроде "вза-

\footnotetext{
1) Тот факт, что любой связный граф содержит в качестве своего связного подграффа дерево, использовался, например, в работе [31] при перечислении связных помеченных графов с данным цикломатическим числом и в работах [9], [10], [32] для оценки сверху скорости убывания многоточечных корреляций. В [33] строится классификация связных непомеченных графов, в которой понятие дерева используется для формулировки алгоритма построения более сложных графов из данных графов. При этом, однако, рассматриваемое дерево не является каким-либо подграфом строящихся графов. В [34] деревья использовались для оценки числа 2-связных множеств с заданной мощностью границы в двудольных графах. Но автору неизвестны работы, в которых ранее статьи [28] ставился бы явно и решался вопрос о классификации связных графов с данным множеством помеченных вершин при помощи деревьев с тем же множеством вершин.
} 
имного уничтожения" слагаемых противоположного знака путем теоретического анализа. В результате получается представление $n$-го коэффициента в виде суммы интегралов, маркируемых деревьями, где суммирование производится по всем помеченным деревьям с множеством вершин $V_{n}$. Эта сумма называется древесной суммой.

В частности, если потенциал парного взаимодействия является неотрицательным, то все ненулевые слагаемые в древесной сумме имеют один и тот же знак, совпадающий со знаком числа $(-1)^{n-1}$, т.е. в этом частном случае слагаемые противоположного знака уничтожаются полностью.

Сама по себе идея изменения порядка суммирования, разумеется, не нова и часто используется при исследовании рядов. Так, например, на изменении порядка суммирования основаны преобразования рядов в работах [12]-[27]. Проблема, однако, заключается в том, как при помоши изменения порядка суммирования добиться поставленной цели. К сожалению, во всех переразложениях, сделанных в работах [12]-[27], проблема асимптотической катастрофы не решается.

Наиболее близким к примененному в работе [28] способу изменения порядка суммирования, основанному на древесной классификации связных графов, является способ, использованный в работе [32]. Однако, в отличие от [28], в работе [32] была поставлена задача оценки сверху рассматриваемой суммы, а не получение для нее более простого представления, содержашего меньшее число слагаемых. С целью оценки сверху каждому помеченному дереву $t$ в [32] ставится в соответствие не множество $F(t)$, а множество, состоящее из всех помеченных графов, являющихся надграфами дерева $t$, и, следовательно, содержащее множество $F(t)$. Поэтому в указанной работе, в отличие от [28], каждый связный помеченный граф входит не в одно, а во все множества, маркируемые деревьями, являющимися подграфами этого графа, а изменение порядка суммирования, приводящее к получению нужной авторам оценки, сопровождается еше и добавлением новых слагаемых в сумму.

Метод древесных сумм применим не только для получения оценок величины коэффициентов разложений по активности. Выше объяснялось, как он применяется для получения свободных от асимптотической катастрофы представлений коэффициентов разложений давления и плотности по активности, а в работах [35], [36] (см. также [29]) он использован для построения свободных от асимптотической катастрофы представлений коэффициентов разложений по активности усеченных функций распределения (усеченных корреляционных функций). В работах [37], [38] методом древесных сумм построены свободные от асимптотической катастрофы представления коэффициентов разложения удельного объема в ряд Лорана по степеням активности. Наконец, метод древесных сумм упрошает аналитические исследования [38], [39] указанных степенных рядов. Эти исследования еще более упрошаются, если парный потенциал является неотрицательным и, следовательно, все ненулевые слагаемые в древесной сумме, представляющей $n$-й коэффициент степенного ряда, имеют один и тот же знак. Именно этот случай был рассмотрен в работе [39].

Построенное в данной статье представление вириальных коэффициентов удалось получить, распространив на множество помеченных неразделимых [3] (см. также прило- 
жение А) графов (блоков) тот же принцип классификации, который положен в основу древесной классификации связных помеченных графов. Этот принцип состоит в следуюшем [40]. Для данного множества $\mathfrak{G}(V)$ графов с множеством вершин $V$ строится некоторое его подмножество $\widetilde{\mathfrak{G}}(V) \subset \mathfrak{G}(V)$ мошности, значительно меньшей, чем мошность данного множества $\mathfrak{G}(V)$. Каждому графу $\widetilde{G} \in \widetilde{\mathfrak{G}}(V)$ ставится в соответствие множество $X_{\text {ad }}(\widetilde{G})$, состоящее из некоторых ребер, соединяющих две несмежные в графе $\widetilde{G}$ вершины. Затем каждому графу $\widetilde{G} \in \widetilde{\mathfrak{G}}(V)$ ставится в соответствие множество $\mathfrak{G}(\widetilde{G})$, состояшее из графа $\widetilde{G}$ и всех его надграфов, которые получаются из графа $\widetilde{G}$ добавлением ребер из множества $X_{\text {ad }}(\widetilde{G})$. Если при этом каждый графи из множества $\mathfrak{G}(V)$ входит в одно и только в одно множество вида $\mathfrak{G}(\widetilde{G})$, то в результате получается разбиение множества графов $\mathfrak{G}(V)$ на непересекающиеся множества, т.е. классы, маркируемые графами из множества $\widetilde{\mathfrak{G}}(V)$. А множество графов $\mathfrak{G}(V)$ представляется в виде объединения непересекающихся множеств:

$$
\mathfrak{G}(V)=\bigcup_{\widetilde{G} \in \widetilde{\mathfrak{G}}(V)} \mathfrak{G}(\widetilde{G}) .
$$

При этом граф $\widetilde{G}$, служаший меткой класса графов $\mathfrak{G}(\widetilde{G})$, называется каркасом этих графов. Классификация такого рода называется каркасной классификацией [40].

Описание каркасной классификации помеченных блоков ${ }^{2}$ оказалось значительно сложнее, чем описание древесной классификации связных помеченных графов, приведенное в работе [28]. В самом деле, в древесной классификации связных помеченных графов метками классов (каркасами) являются помеченные деревья. Понятие помеченных деревьев является достаточно простым и широко известным в математике. Поэтому при введении древесной классификации связных помеченных графов не было никакой необходимости напоминать читателю определение дерева.

Иначе обстоит дело при описании классификации помеченных блоков. Здесь приходится объяснять, как устроены метки классов. В качестве меток классов, на которые разлагается множество помеченных блоков, выступают наборы простых циклов, удовлетворяюших определенным в приложении Б условиям. Такие наборы простых циклов называются ансамблями каркасных циклов. А каркасом класса блоков, маркируемого данным ансамблем каркасных циклов, является граф, который может быть представлен в виде объединения [3] (см. также приложение А) циклов, составляющих данный ансамбль каркасных циклов. Такие объекты в теории графов ранее не вводились и не исследовались. Их определение является достаточно сложным и составляет значительную часть приложения Б.

Общее представление об этой классификации, без описания полной непротиворечивой системы математических определений, дано в разделе 2.

В разделе 3 на основе каркасной классификации помеченных блоков получено представление $n$-го вириального коэффициента в виде суммы интегралов, в которой число

\footnotetext{
${ }^{2)}$ Впервые о создании этой классификации заявлено в [40]. Ее краткое изложение (без обоснований) дано в [41] и ниже в настоящей статье.
} 
слагаемых равно не числу всех помеченных блоков с множеством вершин $V_{n}$, а существенно меньшему числу классов, на которые разлагается это множество блоков.

В разделе 4 дано формальное определение асимптотической катастрофы в представлениях коэффициентов степенных рядов и объяснена значимость этого определения; введена древесная классификация определенного в приложении Б множества ансамблей каркасных циклов и доказано, что полученные в разделе 3 представления вириальных коэффициентов свободны от явления асимптотической катастрофы, понимаемой в смысле определения, данного в этом разделе.

В приложении А определены основные понятия теории графов, используемые в статье. В нем дано также определение множества $X_{\mathrm{ad}}(t)$ ребер, допустимых для данного дерева $t$ с множеством вершин $V_{n}$. В приложении Б дано описание системы математических определений, на которую опирается каркасная классификация помеченных блоков. В нем определено упорядочение простых циклов с помеченными вершинами, лежащее в основе каркасной классификации помеченных блоков. Здесь также определены множества ансамблей каркасных циклов для всех классов блоков с $n$ помеченными вершинами и допустимых ребер ансамбля каркасных циклов.

\section{2. КАРКАСНАЯ КЛАССИФИКАЦИЯ БЛОКОВ}

В настояшем разделе излагаются принципы каркасной классификации двусвязных помеченных графов (помеченных блоков) и даны два определения, проясняющие сходство этих принципов с принципами древесной классификации помеченных графов. Все остальные определения, используемые при построении каркасной классификации блоков, вынесены в приложение Б.

Предполагается, что подлежащее классификации множество $\mathfrak{B}(n)=\{B\}$ состоит из всех блоков с $n$ помеченными вершинами. Без ограничения общности можно считать, что эти вершины помечены натуральными числами, и отождествлять множество этих вершин с множеством $V_{n}=\{1,2, \ldots, n\}$. Так как решение проблемы классификации блоков из множества $\mathfrak{B}(n)$ в случае, когда $n=2$, является тривиальным, то мы в дальнейшем будем рассматривать только классификацию блоков из множества $\mathfrak{B}(n)$, где $n \geqslant 3$. Мы будем называть блоком неразделимый в смысле Харари (см. приложение А) графф с множеством вершин $V_{n}$, где $n \geqslant 3$.

В каркасной классификации помеченных блоков с множеством вершин $V_{n}$, излагаемой в настоящей статье, метками классов являются ${ }^{3)}$ наборы простых циклов [3] (см. также приложение А), удовлетворяющие определенным в приложении Б условиям. Эти наборы циклов называются ансамблями каркасных ииклов и образуют множество, которое мы обозначим через $\mathfrak{C}(n)=\{\mathbf{C}\}$.

Объединение [3] (см. также приложение А) всех циклов из ансамбля циклов $\mathbf{C} \in \mathfrak{C}(n)$ обозначим $S(\mathbf{C})$. Граф $S(\mathbf{C})$ является блоком при любом $\mathbf{C} \in \mathfrak{C}(n)$. Каждому ансамб-

\footnotetext{
3) Доказательство этого и других подобных категорических утверждений ради простоты изложения не приводятся. Полное изложение каркасной классификации блоков будет опубликовано отдельно.
} 
лю циклов $\mathbf{C} \in \mathfrak{C}(n)$ ставится в соответствие множество $X_{\mathrm{ad}}(\mathbf{C})$ допустимых ребер этого ансамбля, удовлетворяющих определенным условиям (см. приложение Б). Для каждого ансамбля циклов $\mathbf{C} \in \mathfrak{C}(n)$ определено множество $\mathfrak{B}(\mathbf{C})$ блоков, помеченное этим ансамблем циклов и состоящее из блока $S(\mathbf{C})$ и всех блоков, которые можно получить из блока $S(\mathbf{C})$ добавлением [3] (см. также приложение А) ребер из множества допустимых ребер $X_{\mathrm{ad}}(\mathbf{C})$ данного ансамбля циклов $\mathbf{C}$.

ОПРЕДЕЛЕНИЕ 1. Блок $S(\mathbf{C})$ называется каркасом блоков из множества $\mathfrak{B}(\mathbf{C})$.

Если $\mathbf{C}$ и $\mathbf{C}^{\prime}$ - два различных ансамбля из множества $\mathfrak{C}(n)$, то множества $\mathfrak{B}(\mathbf{C})$ и $\mathfrak{B}\left(\mathbf{C}^{\prime}\right)$ не пересекаются. Более того, каждый блок $B \in \mathfrak{B}(n)$ принадлежит одному и только одному из множеств вида $\mathfrak{B}(\mathbf{C})$. Отсюда следует, что множество блоков $\mathfrak{B}(n)$ может быть представлено в виде разложения

$$
\mathfrak{B}(n)=\bigcup_{\mathbf{C} \in \mathfrak{C}(n)} \mathfrak{B}(\mathbf{C})
$$

на непересекаюшиеся множества, т.е. классы, маркируемые ансамблями циклов из множества $\mathfrak{C}(n)$.

Ансамбли циклов из множества $\mathfrak{C}(n)$ находятся во взаимно однозначном соответствии с каркасами. Поэтому наряду с ансамблем циклов $\mathbf{C} \in \mathfrak{C}(n)$ роль метки класса блоков $\mathfrak{B}(\mathbf{C})$ может играть каркас $S(\mathbf{C})$.

ОПРеДЕЛЕниЕ 2 . Граф $B_{\max }(\mathbf{C})$, получающийся добавлением к каркасу $S(\mathbf{C})$ множества ребер $X_{\mathrm{ad}}(\mathbf{C})$, называется максимальным надграфом этого каркаса.

Из определения класса блоков $\mathfrak{B}(\mathbf{C})$ следует, что любой блок из класса $\mathfrak{B}(\mathbf{C})$ является подграфом максимального надграффа $B_{\max }(\mathbf{C})$ каркаса $S(\mathbf{C})$. Таким образом, во введенной классификации помеченных блоков роль каркасов $S(\mathbf{C})$ аналогична роли помеченных деревьев, а роль максимальных надграфов $B_{\max }(\mathbf{C})$ каркасов $S(\mathbf{C})$ аналогична роли максимальных надграфов помеченных деревьев в древесной классификации [28] связных помеченных графов.

\section{3. ПРЕДСТАВЛЕНИЯ ВИРИАЛЬНЫХ КОЭФФИЦИЕНТОВ КАРКАСНЫМИ СУММАМИ}

В этом и следуюшем разделах рассматриваются системы классических частиц в $\nu$-мерном действительном евклидовом пространстве $\mathbb{R}^{\nu}$ с парным взаимодействием, характеризуемым потенциалом парного взаимодействия $\Phi(\mathbf{r})$, где $\mathbf{r}=\left(r^{(1)}, r^{(2)}, \ldots\right.$ $\left.\ldots, r^{(\nu)}\right) \in \mathbb{R}^{\nu}$.

Как обычно, введем функцию Майера [1], [8]

$$
f_{i j}=e^{-\beta \Phi\left(\mathbf{r}_{i}-\mathbf{r}_{j}\right)}-1
$$

где $\beta=1 / k T, k$ - постоянная Больцмана, $T$ - абсолютная температура. Обозначим $X(B)$ - множество ребер блока $B,\{u, v\}$ - ребро, инцидентное вершинам $u$ и $v, \Lambda \subset \mathbb{R}^{\nu}-$ 
ограниченное измеримое множество в $\nu$-мерном действительном евклидовом пространстве $\mathbb{R}^{\nu},|\Lambda|$ - мера Лебега множества $\Lambda$.

При малых значениях плотности $\rho$ термодинамический предел давления $p$ представляется в виде ряда по степеням плотности [1], [8], [9]:

$$
p=p(\rho)=\beta^{-1}\left(\rho+\sum_{n=2}^{\infty} B_{n} \rho^{n}\right) .
$$

В этом разложении вириальные коэффициенты $B_{n}$ традиционно определяются формулой

$$
B_{n}=\sum_{B \in \mathfrak{B}(n)}-\frac{n-1}{n !} \lim _{\Lambda \rightarrow \infty}|\Lambda|^{-1} \int_{\Lambda^{n}} \prod_{\{u, v\} \in X(B)} f_{u v}(d \mathbf{r})_{n}
$$

где

$$
\begin{aligned}
(d \mathbf{r})_{n} & =d \mathbf{r}_{1} d \mathbf{r}_{2} \ldots d \mathbf{r}_{n}, \\
d \mathbf{r}_{i} & =d r_{i}^{(1)} d r_{i}^{(2)} \ldots d r_{i}^{(\nu)} .
\end{aligned}
$$

Традиционные представления вириальных коэффициентов по формуле (4) приводят к асимптотической катастрофе [4]-[6]. Цель этого раздела - найти представления вириальных коэффициентов, свободные от явления асимптотической катастрофы.

ТЕОрема 1. При $n \geqslant 2$ вириальнье коэффициенты $B_{n}$ могут быть представленbl в виде

$$
B_{n}=\sum_{\mathbf{C} \in \mathfrak{C}(n)}-\frac{n-1}{n !} J(\mathbf{C}),
$$

əде

$$
J(\mathbf{C})=\lim _{\Lambda \rightarrow \infty}|\Lambda|^{-1} \int_{\Lambda^{n}} \prod_{\{u, v\} \in X(S(\mathbf{C}))} f_{u v} \prod_{\{\tilde{u}, \tilde{v}\} \in X_{\mathrm{ad}}(\mathbf{C})} e^{-\beta \Phi\left(\mathbf{r}_{\tilde{u}}-\mathbf{r}_{\tilde{v}}\right)}(d \mathbf{r})_{n} .
$$

ДокАЗАТЕЛьСТво. Используя разложение $(1)$ множества $\mathfrak{B}(n)$ на непересекаюшиеся подмножества, из формулы (4) получаем

$$
\begin{aligned}
B_{n} & =-\frac{n-1}{n !} \sum_{B \in \mathfrak{B}(n)} \lim _{\Lambda \rightarrow \infty}|\Lambda|^{-1} \int_{\Lambda^{n}} \prod_{\{u, v\} \in X(B)} f_{u v}(d \mathbf{r})_{n}= \\
& =-\frac{n-1}{n !} \sum_{\mathbf{C} \in \mathfrak{C}(n)} \sum_{B \in \mathfrak{B}(\mathbf{C})} \lim _{\Lambda \rightarrow \infty}|\Lambda|^{-1} \int_{\Lambda^{n}} \prod_{\{u, v\} \in X(B)} f_{u v}(d \mathbf{r})_{n} .
\end{aligned}
$$

Совокупность блоков $\mathfrak{B}(\mathbf{C})$ представляет собой совокупность блоков, состоящую из блока $S(\mathbf{C})$ и всех надграфов этого блока, получаюшихся добавлением к блоку $S(\mathbf{C})$ 
одного или нескольких ребер из множества допустимых ребер $X_{\text {ad }}(\mathbf{C})$. Отсюда вытекает равенство

$$
\begin{aligned}
& \sum_{B \in \mathfrak{B}(\mathbf{C})} \lim _{\Lambda \rightarrow \infty}|\Lambda|^{-1} \int_{\Lambda^{n}} \prod_{\{u, v\} \in X(B)} f_{u v}(d \mathbf{r})_{n}= \\
& \quad=\lim _{\Lambda \rightarrow \infty}|\Lambda|^{-1} \int_{\Lambda^{n}} \prod_{\{u, v\} \in X(S(\mathbf{C}))} f_{u v} \prod_{\{\tilde{u}, \tilde{v}\} \in X_{\mathrm{ad}}(\mathbf{C})} e^{-\beta \Phi\left(\mathbf{r}_{\tilde{u}}-\mathbf{r}_{\tilde{v}}\right)}(d \mathbf{r})_{n} .
\end{aligned}
$$

Подставляя в формулу (9) вместо суммы, стояшей в левой части равенства (10), правую часть этого равенства, мы и получаем утверждение теоремы.

Сумма в правой части равенства (7) называется каркасной суммой.

ЗАмЕчАниЕ. При $n=3$ представление вириального коэффициента формулой (7) проще, чем его представление, данное в работе [6].

\section{4. АСИМПТОТИЧЕСКАЯ КАТАСТРОФА И ЕЕ ПРЕОДОЛЕНИЕ}

В этом разделе мы будем преследовать следуюшие основные цели: 1) ввести формальное определение асимптотической катастрофы и выяснить его значимость; 2) ввести древесную классификацию ансамблей каркасных циклов из множества $\mathfrak{C}(n) ; 3)$ доказать, что полученные в разделе 3 представления вириальных коэффициентов свободны от явления асимптотической катастрофы.

Как было отмечено, традиционные представления коэффициентов степенных рядов, применяемых в классической статистике, приводят к асимптотической катастрофе. Одним из основных ее проявлений является катастрофически быстрый рост с увеличением $n$ ошибки вычисления коэффициента при переменной в степени $n$. Источниками указанного роста ошибки вычислений являются, во-первых, возрастание сложности вычисления отдельных слагаемых суммы, выражающей $n$-й коэффициент, а во-вторых, катастрофически быстрое возрастание с ростом $n$ числа слагаемых в этой сумме. В случае расчета вириальных коэффициентов это приводит к тому, что для реалистических потенциалов удается более или менее точно вычислить не более пяти первых коэффицциентов.

Случай использования метода Монте-Карло для расчета коэффициентов майеровских разложений проанализирован в работе [6], где показано, что при традиционном представлении этих коэффициентов вместе с ростом $n$ катастрофически быстро растут как дисперсия оценки, так и коэффициент вариации оценки $n$-го коэффициента разложений. Поэтому практически неизбежен катастрофически быстрый рост как абсолютной, так и относительной ошибки вычисления $n$-го коэффициента. Ниже будет дано формальное определение асимптотической катастрофы, учитываюшее важность этого ее проявления.

Чтобы объяснить мотивы, повлиявшие на выбор формулировки этого определения, нам потребуется дать формальное определение того, в каком случае рост дисперсии 
оценки методом Монте-Карло коэффициентов степенного ряда считается катастрофически быстрым. Очевидно, что скорость роста при увеличении $n$ дисперсии оценки разумно сопоставлять со скоростью роста $n$-го коэффициента ряда. Так как рассматриваемые в классической статистике ряды имеют конечный ненулевой радиус сходимости, то коэффициенты таких рядов не могут расти быстрее чем экспоненциально. То есть для каждого ряда существует такое число $A$, что величина $A^{n}$ является оценкой сверху $n$-го коэффициента ряда. Поэтому представляется целесообразным считать, что при увеличении $n$ рост дисперсий оценок методом Монте-Карло коэффициентов ряда не является катастрофически быстрым, когда они, так же как и сами коэффициенты ряда, возрастают не быстрее чем экспоненциально.

ОПРЕДЕЛЕНИЕ 3. Дисперсии оценок методом Монте-Карло коэффициентов данного сходящегося степенного ряда не растут катастрофически быстро с ростом $n$, если существует такое число $C>0$, что величина $C^{n}$ является верхней границей дисперсии оценки $n$-го коэффициента этого ряда. В противном случае эти дисперсии растут катастрофически быстро с возрастанием $n$.

Вводимое ниже определение асимптотической катастрофы формулируется исходя из следующих соображений.

Во-первых, оно должно соответствовать описанию асимптотической катастрофы, данному в работах [4], [5]. Иначе говоря, это определение должно удовлетворять следующему условию: всякий раз, когда в представлениях коэффициентов степенного ряда имеет место асимптотическая катастрофа в смысле ее описания, приведенного в работах [4], [5], она имеет место и в смысле данного определения.

Во-вторых, при изучении возможности оценки методом Монте-Карло коэффициентов степенных рядов разумно ограничиться рассмотрением случаев, когда число испытаний, производимых для оценки отдельных слагаемых в сумме, представляющей $n$-й коэффищиент степенного ряда, не превосходит некоторой константы, не зависяшей от $n$ (иначе катастрофического роста дисперсии ошибки при увеличении $n$ можно избежать за счет возрастания объема вычислений). При таком ограничении определение асимптотической катастрофы надо сделать настолько широким, чтобы всякий раз, когда представление коэффициентов ряда не удовлетворяет определению, не было бы оснований полагать, что происходит катастрофический рост дисперсий оценок коэффициентов ряда.

ОпреДЕЛЕниЕ 4. В представлениях коэффициентов степенного ряда присутствует феномен асимптотической катастрофы, если при любом $D>0$ число слагаемых в сумме, представляюшей коэффициент при переменной в степени $n$, при $n \rightarrow \infty$ растет быстрее, чем величина $(n !)^{2} D^{n}$. В противном случае в представлениях коэффициентов степенного ряда феномен асимптотической катастрофы отсутствует.

Таким образом, введенное определение асимптотической катастрофы дает критерий, который позволяет выделить те представления коэффициентов степенного ряда, которые обладают следуюшими двумя недостатками: 1) использование этих представлений 
при оценке методом Монте-Карло коэффициентов данного степенного ряда практически неизбежно приводит с ростом $n$ к катастрофически быстрому росту как дисперсии оценки коэффищиента при переменной в степени $n$, так и (в случае сходящегося степенного ряда) коэффициента вариации этой оценки; 2) эти представления крайне затрудняют аналитическое исследование представленных ими коэффициентов и, в частности, оценку радиуса сходимости степенного ряда с этими коэффициентами.

Более того, это определение асимптотической катастрофы дает нам ориентир для построения таких представлений коэффициентов степенных рядов, которые лишены указанных выше двух недостатков.

Согласно определению 4 в традиционных представлениях коэффициентов майеровских разложений феномен асимптотической катастрофы присутствует, так как при $n \rightarrow \infty$ число слагаемых в сумме, представляюшей коэффициент при переменной в степени $n$, растет как $2^{n(n-1) / 2}$, т.е. значительно быстрее, чем величина $(n !)^{2} D^{n}$.

Напротив, полученные в [28] представления древесными суммами коэффициентов разложений давления и плотности по активности свободны от явления асимптотической катастрофы, понимаемой как в смысле ее описания, данного в работах [4], [5], так и в смысле определения 4 .

Возможность оценки методом Монте-Карло древесных сумм, представляющих коэффициенты разложений по активности, была исследована в работе [6] (см. также [29]) для случая регулярного [2] и неотрицательного потенциала парного взаимодействия. При этом предполагалось, что число испытаний, производимых для оценки каждого слагаемого рассматриваемой древесной суммы, является величиной постоянной, не зависяшей от $n$. Установлено, что как дисперсия оценки $n$-го коэффициента, так и коэффициент вариации этой оценки при $n \rightarrow \infty$ не только не растут катастрофически быстро, но и стремятся к нулю.

Докажем, что в представлениях вириальных коэффициентов в виде каркасных сумм (7) явление асимптотической катастрофы отсутствует. С этой целью введем классификацию ансамблей каркасных циклов из множества $\mathfrak{C}(n)^{4)}$. Для этого нам потребуется ввести следуюшие обозначения: $\beta_{1}(C)$ - меньшая из двух вершин, смежных с вершиной $\alpha(C)$ (см. приложение Б) в простом цикле $C ; \beta_{2}(C)$ - другая вершина цикла $C$, смежная с вершиной $\alpha(C)$ в этом цикле.

ОПРеДЕЛЕНИЕ 5. Ребро цикла $C(0)$ из ансамбля $\mathbf{C} \in \mathfrak{C}(n)$, соединяюшее вершины $\alpha=1$ и $\beta_{2}(C(0))$, называется выделенным ребром цикла $C(0)$ из ансамбля $\mathbf{C .}$

Если цикл $C(i)_{q}$, где $q \geqslant 1$, принадлежит ансамблю $\mathbf{C} \in \mathfrak{C}(n)$, то ребро этого цикла, соединяюшее вершину $\gamma\left((i)_{q}, m\left((i)_{q} ; \mathbf{C}\right) ; \mathbf{C}\right)$ и непосредственно предшествуюшую ей по этому циклу вершину, называется выделенным ребром этого цикла.

\footnotetext{
4) Определения ансамбля циклов и множества $\mathfrak{C}(n)$ ансамблей каркасных циклов, способ нумерации (маркирования) циклов из данного ансамбля циклов, а также определения вершины, непосредственно следующей по данному циклу за данной его вершиной, и вершины $\gamma\left((i)_{q}, m\left((i)_{q} ; \mathbf{C}\right) ; \mathbf{C}\right)$ см. в приложении Б.
} 
ОПредЕлЕниЕ 6 . Множество выделенных ребер всех циклов ансамбля $\mathbf{C}$ называется выделенным множеством ребер этого ансамбля.

ОПРЕДЕЛЕНИЕ 7. Граф̆ $t(S(\mathbf{C}))$, получающийся из блока $S(\mathbf{C})$, где $\mathbf{C} \in \mathfrak{C}(n)$, удалением выделенного множества ребер ансамбля $\mathbf{C}$, называется древеснылм образом ансамбля $\mathbf{C}$.

Очевидно, что множество всех вершин графиа $t(S(\mathbf{C}))$ совпадает с множеством вершин блока $S(\mathbf{C})$, а граф $t(S(\mathbf{C}))$ является связным и представляет собой дерево.

Совокупность всех ансамблей $\mathbf{C}$, удовлетворяющих условию $t(S(\mathbf{C}))=t$, обозначим $\mathfrak{C}(t)$.

Множество древесных образов всех ансамблей циклов из множества $\mathfrak{C}(n)$ обозначим $T_{i}(n)$. Если $t$ и $t^{\prime}$ - два различных древесных образа из множества $T_{i}(n)$, то, очевидно, $\mathfrak{C}(t) \bigcap \mathfrak{C}\left(t^{\prime}\right)=\varnothing$. Поэтому множество $\mathfrak{C}(n)$ ансамблей каркасных циклов может быть представлено в виде разложения

$$
\mathfrak{C}(n)=\bigcup_{t \in T_{i}(n)} \mathfrak{C}(t)
$$

на непересекающиеся множества, т.е. классы. Мы получили древесную классификацию множества $\mathfrak{C}(n)$ ансамблей каркасных циклов, в которой меткой класса служит дерево, которое для каждого ансамбля циклов С из этого класса является остовным подграфом объединения $S(\mathbf{C})$ всех циклов ансамбля $\mathbf{C}$.

Из полученного разложения $(11)$ множества $\mathfrak{C}(n)$ ансамблей каркасных циклов вытекает

ТЕОРема 2. Число ансамблей в совокупности $\mathfrak{C}(n)$ не превосходит числа $(n-1)^{n-3}(n-2)^{n-2}$.

ДокАЗАтЕльСтво. Рассмотрим дерево $t$, удовлетворяющее условию, что совокупность $\mathfrak{C}(t)$ не является пустым множеством. Из определения каркасного цикла следует, что все ансамбли из совокупности $\mathfrak{C}(t)$ имеют общий цикл $C(0)$. При этом цепь, получающаяся из цикла $C(0)$ удалением выделенного ребра $\left\{\alpha, \beta_{2}(C(0))\right\}$, совпадает с цепью, соединяюшей в дереве $t$ вершину $\alpha=1$ с вершиной $\beta_{2}(C(0))$. Эта цепь удовлетворяет условию, что если вершина $v$ непосредственно следует за вершиной $u$ при движении по этой цепи от вершины $\alpha=1$, а отличная от вершины $v$ вершина $v^{\prime}$ является вершиной, смежной с вершиной $u$ и следуюшей за вершиной $u$ при движении от вершины $\alpha=1 \mathrm{k}$ вершине $v^{\prime}$ по ребрам дерева $t$, то имеет место неравенство $v<v^{\prime}$. Это условие позволяет единственным образом определить вьшеупомянутую цеп дерева $t$ и, следовательно, ее концевые вершины. В каждом блоке $S(\mathbf{C})$, где $\mathbf{C} \in \mathfrak{C}(t)$, концевые вершины этой цепи соединяются ребром $\left\{\alpha(C), \beta_{2}(C(0))\right\}$. Так как вершина $\beta_{1}(\mathbf{C})$ не может быть инцидентной выделенному ребру какого-либо цикла $C$ из ансамбля $\mathbf{C} \in \mathfrak{C}(t)$ и, следовательно, не является висячей вершиной дерева $t$, число остальных висячих вершин этого дерева (т.е. не совпадающих ни с вершиной $\beta_{2}(C(0))$, ни с вершиной $\alpha=1$ ) не превосходит числа $n-3$. 
Итак, общий цикл $C(0)$ ансамблей из совокупности $\mathfrak{C}(t)$, а следовательно, и вершины $\beta_{1}(C(0))$ и $\beta_{2}(C(0))$ однозначно определяются деревом $t$. Обозначим эти вершины соответственно $\beta_{1}(t)$ и $\beta_{2}(t)$.

Рассмотрим висячую вершину $v$ дерева $t$, не совпадаюшую ни с вершиной $\alpha=1$, ни с вершиной $\beta_{2}(t)$. Каков бы ни был ансамбль $\mathbf{C}$ из совокупности $\mathfrak{C}(t)$, вершина $\beta_{1}(t)$ по определению не может следовать за вершиной $v$ ни по одному из циклов этого ансамбля. Следовательно, число вершин дерева $t$, которые могли бы быть следуюшими за вершиной $v$ по какому-нибудь из циклов ансамбля $\mathbf{C}$ из совокупности $\mathfrak{C}(t)$, не превосходит числа $n-2$. Отсюда вытекает, что число ансамблей в совокупности $\mathfrak{C}(t)$ не превосходит числа $(n-2)^{n-3}$.

Каждое дерево, являющееся древесным образом хотя бы одного ансамбля циклов $\mathbf{C}$ из совокупности $\mathfrak{C}(n)$, представляет собой помеченное дерево $t$ с $n$ вершинами, в котором вершина $\alpha=1$ является концевой, причем смежная с ней вершина $\beta_{1}(t)$ не может совпадать с вершиной $n$. Число таких деревьев не превосходит числа $(n-2)(n-1)^{n-3}$. Отсюда следует, что число всех ансамблей совокупности $\mathfrak{C}(n)$ не превосходит числа $(n-1)^{n-3}(n-2)^{n-2}$, что и требовалось доказать.

Теорема 2 дает довольно грубую оценку сверху числа слагаемых в каркасной сумме, представляющей по формуле (7) вириальный коэффициент $B_{n}$. Тем не менее из этой оценки становится очевидным, что число слагаемых в каркасной сумме, представляющей вириальный коэффициент $B_{n}$, сушественно меньше, чем в сумме, традиционно представляюшей этот коэффициент по формуле (4) (см. также табл. 2), а сложность вычисления отдельных слагаемых не увеличивается. Поэтому представление вириальных коэффициентов каркасными суммами по формуле (7) более удобно, чем их традиционное представление по формуле (4).

Из теоремы 2 с помошью формулы Стирлинга после элементарных преобразований получаем

СлЕДСТвИЕ 1. Число ансамблей простых ииклов в совокупности $\mathfrak{C}(n)$ не превосходит числа $[(n-2) !]^{2} e^{2 n} /\left(2 \pi e^{3}(n-1)^{3 / 2}(n-2)^{1 / 2}\right)$.

Из следствия 1 вытекает, что представления вириальных коэффициентов формулой (7) свободны от явления асимптотической катастрофы как в смысле ее описания, данного в работах [4], [5], так и в смысле определения 4.

В случае регулярного и неотрицательного потеншиала парного взаимодействия из следствия 1 вытекает

СлЕДСтвИЕ 2. Пусть потенциал парного взаимодействия является регулярныцм и неотрицательным, а при оценке методом Монте-Карло вириальных коәффициентов используется их представление в виде каркасных сумм по формуле (7). Тогда дисперсия $\sigma^{2}\left(B_{n}\right)$ оценки вириального коэффициента $B_{n}$ не растет катастрофически бъстро с ростом $n$, если только для оченки каждого из слагаемых каркасной суммы производится одно и то же число испытаний. 
ДокАЗАТЕЛЬСтво. В случае регулярного и неотрицательного потенциала парного взаимодействия функция Майера $f(\mathbf{r})=e^{-\beta \Phi(\mathbf{r})}-1$ является неположительной. Кроме того, модуль этой функции, а также больцмановская функция $e^{-\beta \Phi(\mathbf{r})}$ удовлетворяют неравенствам

$$
0 \leqslant|f(\mathbf{r})|, e^{-\beta \Phi(\mathbf{r})} \leqslant 1
$$

Пусть $N$ - число испытаний, производимых для оценки методом Монте-Карло величины $J(\mathbf{C})$, определенной формулой (8). Тогда из формулы (12) нетрудно вывести следуюшее неравенство для дисперсии $\sigma^{2}(J(\mathbf{C}))$ оценки методом Монте-Карло величины $J(\mathbf{C})$ :

$$
\sigma^{2}(J(\mathbf{C})) \leqslant \frac{(C(\beta))^{n-1}}{4 N},
$$

где

$$
C(\beta)=\int_{\mathbb{R}^{\nu}}|f(\mathbf{r})| d \mathbf{r} .
$$

Из условий следствия 2, оценки (13) и следствия 1 получаем следуюшее неравенство для дисперсии $\sigma^{2}\left(B_{n}\right)$ оценки методом Монте-Карло вириального коэффициента $B_{n}$ :

$$
\sigma^{2}\left(B_{n}\right) \leqslant \frac{1}{8 N \pi e^{3}} \frac{e^{2 n}(C(\beta))^{n-1}}{n^{2}(n-1) \sqrt{(n-1)(n-2)}} .
$$

Из этого неравенства становится очевидным, что согласно определению 3 дисперсия $\sigma^{2}\left(B_{n}\right)$ оценки методом Монте-Карло вириального коэффициента $B_{n}$ не растет катастрофически быстро с ростом $n$, если только для оценки каждого из слагаемых каркасной суммы производится одно и то же число испытаний. Что и требовалось доказать.

По-видимому, и в более общем случае устойчивого регулярного потенциала утверждение следствия 2 остается верным.

\section{ПРИЛОЖЕНИЕ А}

В статье используется ряд понятий теории графов. К сожалению, в теории графов нет единой терминологии. В данной статье используется терминология, введенная в книге [3], которой придерживается большая часть специалистов по теории графов. Для удобства читателей в данном приложении приводятся определения понятий теории графов, используемых в статье. Вводится также определение множества $X_{\mathrm{ad}}(t)$ допустимых ребер дерева $t$. Большая часть содержания этого приложения заимствована из книги [3].

Граф называется помеченным ( перенумерованным ), если его вершины отличаются одна от другой какими-либо пометками, например числами $1,2, \ldots, n$.

Маршрутом в графе $G$ называется чередуюшаяся последовательность его вершин и ребер; эта последовательность начинается вершиной и заканчивается вершиной, а каждое ребро этой последовательности инцидентно двум вершинам, одна из которых непосредственно предшествует этому ребру, а другая непосредственно за ним следует. Количество ребер в маршруте называется его длиной. 
Маршрут называется цепью, если все его ребра различны. Маршрут называется простой цепью, если все его вершины (а следовательно, и все его ребра) различны.

Маршрут замкнут, если его первая вершина совпадает с его последней вершиной. Замкнутый маршрут называется простым ииклом, если все его $n$ вершин различны и $n \geqslant 3$.

Очевидно, что простой цикл является цепью. Граф, множество вершин которого совпадает с множеством вершин данной цепи, а множество ребер совпадает с множеством ребер этой цепи, можно отож дествлять с данной цепю. В частности, граф̆, множество вершин которого совпадает с множеством вершин данного простого цикла, а множество ребер совпадает с множеством ребер этого цикла, можно отождествлять с данным циклом.

Граф̆ называется связныц м если любая пара его вершин соединена в этом графе простой цепью.

Граф̆ называется полным, если каждая пара его вершин является смежной в этом графе.

Максимальный связный подграф̆ графа $G$ называется его компонентой связности или просто компонентой графа $G$.

Удаление вершины $v$ из графа $G$ приводит к его подграфу $G-v$, содержашему все вершины графа $G$, за исключением вершины $v$, и все ребра графа $G$, не инцидентные вершине $v$. Другими словами, граф $G-v$ есть максимальный подграф графа $G$, не содержащий вершины $v$.

Точкой сочленения связного графа называется вершина, удаление которой приводит к распадению этого графа на компоненты связности. Неразделимым (в смысле Харари) графом (блоком) называется имеюший не менее двух вершин, связный, без точек сочленения граф.

Объединением $G_{1} \cup G_{2}$ графа $G_{1}$ с множеством вершин $V_{1}$ и множеством ребер $X_{1}$ и графа $G_{2}$ с множеством вершин $V_{2}$ и множеством ребер $X_{2}$ называется граф с множеством вершин $V=V_{1} \cup V_{2}$ и множеством ребер $X=X_{1} \cup X_{2}$.

Рассмотрим множество $T_{n}=\{t\}$ деревьев с множеством вершин $V_{n}$ и корнем $\alpha=1$. Пусть $t \in T_{n}-$ некоторое дерево из множества $T_{n}$. Введем следующие обозначения: $V(t, h)$ - слой вершин дерева $t$, находяшихся на высоте $h ; Z(t)$ - множество ребер, каждое из которых соединяет две вершины дерева $t$, принадлежашие одному и тому же слою дерева $t ; H(t)$ - высота дерева $t$.

Напомним, что множество $V_{n}$, являюшееся множеством вершин любого дерева $t \in$ $T_{n}$, состоит из первых $n$ чисел натурального ряда. Мы будем говорить, что вершина $v \in V_{n}$ следует в множестве $V_{n}$ за вершиной $u \in V_{n}$, если $u<v$.

Если вершина $v$ принадлежит слою $V(t, h)$, где $h \geqslant 2$, то через $u(t, v)$ обозначим вершину слоя $V(t, h-1)$, смежную с вершиной $v$ в дереве $t$.

Следуя [28], введем еше ряд обозначений: $Y(t, v)$ - множество всех ребер, соединяюших вершину $v$ из слоя $V(t, h)$, где $h \geqslant 2$, с одной из вершин слоя $V(t, h-1)$, следуюших 
за вершиной $u(t, v)$ в множестве вершин $V_{n}$;

$$
Y_{1}(t, h)=\bigcup_{v \in V(t, h)} Y(t, v) ; \quad Y_{2}(t)=\bigcup_{h=2}^{H(t)} Y_{1}(t, h) ; \quad X_{\mathrm{ad}}(t)=Z(t) \cup Y_{2}(t)
$$

Множество $X_{\text {ad }}(t)$ назовем множеством допустимых ребер дерева $t$.

\section{ПРИЛОЖЕНИЕ Б}

Введем понятие упорядочения циклов с помеченными вершинами. В каркасной классификации множества $\mathfrak{B}(n)$ блоков с $n$ помеченными вершинами, где $n \geqslant 3$, это упорядочение служит основой для определения множества $\mathfrak{C}(n)$ ансамблей каркасных циклов. Далее определим множество $\mathfrak{C}(n)=\{\mathbf{C}\}$ ансамблей каркасных циклов и множество $X_{\text {ad }}(\mathbf{C})$ допустимых ребер ансамбля циклов $\mathbf{C}$, играюших основную роль в определении каркасной классификации множества помеченных блоков $\mathfrak{B}(n)$. Ребро, инцидентное вершинам $u$ и $v$, обозначается $\{u, v\}$.

Мы будем отождествлять простой цикл полного графа с множеством вершин $V_{n}$, где $n \geqslant 3$, и помеченный граф, у которого множество вершин совпадает с множеством вершин этого цикла, а множество ребер - с множеством ребер этого цикла, т.е. будем рассматривать такой простой цикл как граф.

Введем следуюшие обозначения: $V(C)$ - множество вершин простого цикла $C ; \alpha(C)$ - наименьшая из вершин простого цикла $C ; d(C)$ - длина простого цикла $C ; v_{h}(C)-$ вершина, которая соединяется с вершиной $\alpha(C)$ простой цепю простого цикла $C$, имеющей длину $h$ и содержашей вершину $\beta_{1}(C)$ (см. раздел 4$)$. Если вершина $w$ принадлежит множеству вершин цикла $C$, то через $P(C ; w)$ обозначим простую цепь цикла $C$, содержашую вершину $\beta_{1}(C)$ и соединяющую вершины $\alpha(C)$ и $w$.

ОПРеДЕЛЕНИЕ П1. Вершина $v$ называется следующей по простому циклу $C$ за вершиной $u$, если длина простой цепи $P(C ; v)$ больше длины простой цепи $P(C ; u)$.

ОПРЕДЕЛЕНИЕ П2. Вершина $v$ называется непосредственно следующей по простому циклу $C$ за вершиной $u$, если длина простой цепи $P(C ; v)$ на единицу больше длины простой цепи $P(C ; u)$.

Если $C$ и $C^{\prime}$ - два различных простых цикла с вершинами из множества $V_{n}$, то возможны всего четыре случая:

СлучАй 1. Наименьшая из вершин цикла $C$ не совпадает с наименьшей вершиной цикла $C^{\prime}: \alpha(C) \neq \alpha\left(C^{\prime}\right)$.

СлучАй 2. Наименьшая вершина цикла $C$ совпадает с наименьшей вершиной цикла $C^{\prime}: \alpha(C)=\alpha\left(C^{\prime}\right)$, а вершина $\beta_{1}(C)$ цикла $C$ не совпадает с вершиной $\beta_{1}\left(C^{\prime}\right)$ цикла $C^{\prime}$ : $\beta_{1}(C) \neq \beta_{1}\left(C^{\prime}\right)$. 
СлучАй 3. Наименьшая вершина цикла $C$ совпадает с наименьшей вершиной цикла $C^{\prime}: \alpha(C)=\alpha\left(C^{\prime}\right)=\alpha$, а вершина $\beta_{1}(C)$ цикла $C$ совпадает с вершиной $\beta_{1}\left(C^{\prime}\right)$ цикла $C^{\prime}: \beta_{1}(C)=\beta_{1}\left(C^{\prime}\right)=\beta_{1}$. Существует такое натуральное число $h(2 \leqslant h<\min \{d(C)$, $\left.\left.d\left(C^{\prime}\right)\right\}\right)$, что вершина $v_{h}(C)$ не совпадает с вершиной $v_{h}\left(C^{\prime}\right): v_{h}(C) \neq v_{h}\left(C^{\prime}\right)$, тогда как при всех $i=1,2, \ldots, h-1$ вершина $v_{i}(C)$ совпадает с вершиной $v_{i}\left(C^{\prime}\right): v_{i}(C)=v_{i}\left(C^{\prime}\right)$.

Случай 4. Простые циклы $C$ и $C^{\prime}$ имеют различные длины: $d(C) \neq d\left(C^{\prime}\right)$. Наименьшая вершина цикла $C$ совпадает с наименьшей вершиной цикла $C^{\prime}: \alpha(C)=$ $\alpha\left(C^{\prime}\right)=\alpha$. При всех натуральных числах $h$, удовлетворяюших условиям $1 \leqslant h<$ $\min \left\{d(C), d\left(C^{\prime}\right)\right\}$ вершина $v_{h}(C)$ совпадает с вершиной $v_{h}\left(C^{\prime}\right)$.

ОПРЕДЕЛЕНИЕ ПЗ. Простой цикл $C$ предшествует простому циклу $C^{\prime}$ в следуюших четырех случаях: 1) если имеет место случай 1 и $\left.\alpha(C)<\alpha\left(C^{\prime}\right) ; 2\right)$ если имеет место случай 2 и $\left.\beta_{1}(C)<\beta_{1}\left(C^{\prime}\right) ; 3\right)$ если имеет место случай 3 и $\left.v_{h}(C)<v_{h}\left(C^{\prime}\right) ; 4\right)$ если имеет место случай 4 и $d(C)>d\left(C^{\prime}\right)$.

Очевидно, что в множестве всех простых циклов, вершины которых помечены натуральными числами, нет несравнимых элементов относительно введенного определением П3 бинарного отношения. Следовательно, всякое множество простых циклов с вершинами из множества $V_{n}$, на котором установлено введенное определением П3 бинарное отношение, является вполне упорядоченным.

Обозначим через $I$ совокупность, состояшую из числа 0 и всех упорядоченных конечных групп, в которых первым элементом является число 0 , а все остальные элементы являются натуральными числами. Элементы совокупности $I$ будем называть векторами. Число 0 будем рассматривать как нулевой одномерный вектор. Для краткости $(q+1)$-мерный вектор $\left(0, i_{1}, i_{2}, \ldots, i_{q}\right)$ из совокупности $I$ будем обозначать $(i)_{q}$.

ОПРЕДЕЛЕНИЕ П4. Вектор $(i)_{p}$ nредиествует вектору $(j)_{q}$ в следуюших случаях: 1 ) если $p<q$ и имеют место равенства $i_{n}=j_{n}$ при $\left.n=1,2, \ldots, p ; 2\right)$ если $p=q, i_{q}<j_{q}$ и при $n=1,2, \ldots, q-1$ имеют место равенства $i_{n}=j_{n}$. Нулевой одномерный вектор $\mathbf{0}$ предшествует любому другому вектору $(i)_{q}$ из совокупности $I$.

Каков бы ни был вектор $(i)_{q}$ (где $q \geqslant 1$ ) из совокупности $I$, через $(i)_{q-1}$ будем обозначать предшествующий ему $q$-мерный вектор из совокупности $I$. В частности, $(i)_{0}$ обозначает нулевой одномерный вектор $\mathbf{0}$, предшествуюший всем остальным векторам из совокупности $I$.

В настоящей работе рассматриваются наборы простых циклов полного графа с множеством вершин $V_{n}$. Мы будем полагать, что в этих наборах каждому циклу присвоена метка, являюшаяся вектором из совокупности $I$. Цикл, помеченный вектором $(i)_{q} \neq 0$, будем обозначать $C(i)_{q}$. Цикл, помеченный вектором $\mathbf{0}$, будем обозначать $C(0)$.

ОПРЕДЕЛЕНИЕ П5. Вектор $(i)_{q}$, которым помечен простой цикл $C(i)_{q}$, называется вектором-меткой этого цикла.

Множество векторов-меток всех циклов из набора циклов $\mathbf{C}=\left\{C(i)_{q}\right\}$ обозначим $I(\mathbf{C})$. 
ОПРЕДЕЛЕниЕ П6. Набор циклов $\mathbf{C}=\left\{C(i)_{q}\right\}$ называется ансамблем ииклов, если этот набор удовлетворяет следуюшим условиям: 1$)$ в множестве $I(\mathbf{C})$ существует одномерный нулевой вектор-метка $\mathbf{0} ; 2)$ если вектор $(i)_{q}$ входит в множество $I(\mathbf{C})$, то и все векторы, предшествуюшие вектору $(i)_{q}$, также входят в множество $\left.I(\mathbf{C}) ; 3\right)$ если вектор $(i)_{q} \neq 0$ принадлежит множеству векторов-меток $I(\mathbf{C})$, то цикл $C(i)_{q-1}$ предшествует циклу $\left.C(i)_{q} ; 4\right)$ если $(i)_{q},(j)_{q} \in I(\mathbf{C})$ и вектор $(i)_{q}$ предшествует вектору $(j)_{q}$, то цикл $C(i)_{q}$ предшествует циклу $C(j)_{q}$.

Для определения множества $\mathfrak{C}(n)=\{\mathbf{C}\}$ ансамблей циклов и множества $X_{\text {ad }}(\mathbf{C})$ допустимых ребер ансамбля циклов $\mathbf{C}$ нам потребуется ввести ряд обозначений. Пусть $\mathbf{C}=\left\{C(i)_{q}\right\}$ - ансамбль циклов. Через $S\left((i)_{q} ; \mathbf{C}\right)$, где $(i)_{q} \in I(\mathbf{C})$, обозначим объединение [3] (см. также приложение $\mathrm{A}$ ) цикла $C(i)_{q}$ и всех циклов из ансамбля $\mathbf{C}$, помеченных векторами, следуюшими за вектором $(i)_{q}$. Если вектор-метка $(i)_{q}$ отличен от вектора-метки $\mathbf{0}$, то мы полагаем

$$
D\left((i)_{q} ; \mathbf{C}\right)=S\left((i)_{q} ; \mathbf{C}\right)-V\left(C(i)_{q-1}\right),
$$

$\Gamma\left((i)_{q} ; \mathbf{C}\right)$ есть множество вершин цикла $C(i)_{q-1}$, смежных в графе $S\left((i)_{q-1} ; \mathbf{C}\right)$ с вершинами графа $D\left((i)_{q} ; \mathbf{C}\right)$, а $m\left((i)_{q} ; \mathbf{C}\right)$ - мошность множества $\Gamma\left((i)_{q} ; \mathbf{C}\right)$. Вершины множества $\Gamma\left((i)_{q} ; \mathbf{C}\right)$ обозначим $\gamma\left((i)_{q}, 1 ; \mathbf{C}\right), \gamma\left((i)_{q}, 2 ; \mathbf{C}\right), \ldots, \gamma\left((i)_{q}, m\left((i)_{q} ; \mathbf{C}\right) ; \mathbf{C}\right)$.

Если $\alpha\left(C(i)_{q-1}\right) \notin \Gamma\left((i)_{q} ; \mathbf{C}\right)$ и $m\left((i)_{q} ; \mathbf{C}\right) \geqslant 2$, то мы полагаем, что вершины из множества $\Gamma\left((i)_{q} ; \mathbf{C}\right)$ удовлетворяют следуюшему условию: при всех $j$ и $l$, удовлетворяюших неравенствам $1 \leqslant j<l \leqslant m\left((i)_{q} ; \mathbf{C}\right)$, вершина $\gamma\left((i)_{q}, j ; \mathbf{C}\right)$ предшествует вершине $\gamma\left((i)_{q}, l ; \mathbf{C}\right)$ по циклу $C(i)_{q-1}$. Если же $\alpha\left(C(i)_{q-1}\right) \in \Gamma\left((i)_{q} ; \mathbf{C}\right)$ и $m\left((i)_{q} ; \mathbf{C}\right) \geqslant 2$, то мы полагаем, что $\alpha\left(C(i)_{q-1}\right)=\gamma\left((i)_{q}, m\left((i)_{q} ; \mathbf{C}\right) ; \mathbf{C}\right)$, а вершины из множества $\Gamma\left((i)_{q} ; \mathbf{C}\right)$ удовлетворяют следующему условию: при всех $j$ и $l$, удовлетворяющих неравенствам $1 \leqslant j<l \leqslant m\left((i)_{q} ; \mathbf{C}\right)-1$, вершина $\gamma\left((i)_{q}, j ; \mathbf{C}\right)$ предшествует вершине $\gamma\left((i)_{q}, l ; \mathbf{C}\right)$ по циклу $C(i)_{q-1}$.

ОПРЕДЕЛЕниЕ П7. Ребро, связывающее две вершины простого цикла и не принадлежашее этому циклу, называется его диагональю.

Обозначим через $\mathfrak{C}(n)=\{\mathbf{C}\}$ множество ансамблей $\mathbf{C}$ простых циклов, удовлетворяющих следующим условиям:

А. Все вершины циклов этого ансамбля принадлежат множеству вершин $V_{n}$, а каждая вершина из множества $V_{n}$ принадлежит по крайней мере одному из циклов этого ансамбля.

Б. Каждый цикл из ансамбля $\mathbf{C}$ содержит вершину $\alpha=1$.

В. Если ансамбль $\mathbf{C}$ содержит цикл $C(i)_{q}$, где $q \geqslant 1$, то $m\left((i)_{q} ; \mathbf{C}\right) \geqslant 2$, а цикл $C(i)_{q}$ содержит цепь $P\left((i)_{q} ; \mathbf{C}\right)$ цикла $C(i)_{q-1}$, содержащую ребро $\left\{\alpha, \beta_{1}\left(C(i)_{q-1}\right)\right\}$ и соединяюшую вершины $\gamma\left((i)_{q}, m\left((i)_{q} ; \mathbf{C}\right)-1 ; \mathbf{C}\right)$ и $\gamma\left((i)_{q}, m\left((i)_{q} ; \mathbf{C}\right) ; \mathbf{C}\right)$, и не содержит ни одной вершины и ни одного ребра цикла $C(i)_{q-1}$, не принадлежаших цепи $P\left((i)_{q} ; \mathbf{C}\right)$.

Г. При любом $(i)_{q} \in I(\mathbf{C})$ граф $S\left((i)_{q} ; \mathbf{C}\right)$ не содержит ни одного простого цикла, предшествуюшего циклу $C(i)_{q}$. 
Д. При всех $(i)_{q} \in I(\mathbf{C})$, удовлетворяюших условию $(i)_{q} \neq 0$, граф̆ $D\left((i)_{q} ; \mathbf{C}\right)$ связный.

Е. Если два различных вектора $(i)_{q}$ и $\left(i^{\prime}\right)_{q}$, принадлежащих множеству $I(\mathbf{C})$, следуют за одним и тем же вектором $(i)_{q-1}$, то графы $D\left((i)_{q} ; \mathbf{C}\right)$ и $D\left(\left(i^{\prime}\right)_{q} ; \mathbf{C}\right)$ не имеют обших вершин.

Ж. Если ансамбль циклов $\mathbf{C}$ содержит цикл $C(i)_{q}$, где $(i)_{q} \neq 0$, то граф̆ $S\left((i)_{q} ; \mathbf{C}\right)$ не содержит ни одного ребра, являющегося диагональю какого-либо цикла, принадлежашего этому ансамблю и помеченного вектором, предшествуюшим вектору $(i)_{q}$.

Ансамбли циклов, принадлежашие множеству $\mathfrak{C}(n)$, называются ансамблями каркасных ииклов.

Из определения ансамблей каркасных циклов вытекает

СЛЕДСТВИЕ 3. Любой ансамбль каркасных ииклов $\mathbf{C}=\left\{C(i)_{q}\right\}$ удовлетворяет следующему условию: существует вершина $\beta_{1}(\mathbf{C})$, общая для всех ииклов этого ансамбля и являющаяся в каждом из этих ииклов меньшей из двух вериин, смежнихх с верииной $\alpha=1$.

ОПРЕДЕЛЕНИЕ П8. Диагональ $\left\{v_{h}(C), v_{h+k}(C)\right\}$ (где $0 \leqslant h \leqslant d(C)-3$ и $2 \leqslant k<$ $d(C)-h)$ цикла $C$ называется удовлетворяющей условию следования по циклу $C$, если $v_{h+1}(C)<v_{h+k}(C)$.

ОПРЕДЕЛЕНИЕ П9. Ребро $x$ называется допустимым ребром ансамбля циклов $\mathbf{C}=$ $\left\{C(i)_{q}\right\}$, если в этом ансамбле существует цикл $C(i(x))_{q(x)} \in \mathbf{C}$, удовлетворяющий условиям: а) ребро $x$ является диагональю цикла $C(i(x))_{q(x)}$, удовлетворяющей условию следования по этому циклу; б) если вершина $\alpha\left(C(i(x))_{q(x)-1}\right)$ не входит в множество вершин $\Gamma\left((i(x))_{q(x)} ; \mathbf{C}\right)$, то ребро $x$ не относится к числу ребер, которые соединяют одну из вершин цикла $C(i(x))_{q(x)}$, не принадлежашую циклу $C(i(x))_{q(x)-1}$, с одной из вершин, следуюших по циклу $C(i(x))_{q(x)-1}$ за вершиной $\gamma\left((i(x))_{q(x)}, m\left((i(x))_{q(x)} ; \mathbf{C}\right) ; \mathbf{C}\right)$.

Множество всех допустимых ребер ансамбля циклов $\mathbf{C}=\left\{C(i)_{q}\right\}$ обозначим $X_{\mathrm{ad}}(\mathbf{C})$.

Благодарности. Автор выражает искреннюю благодарность И.И. Иванчику за полезные обсуждения проблемы, рассматриваемой в этой работе, и рецензенту за сделанные им ценные замечания. Работа поддержана Российским фондом фундаментальных исследований, грант № 98-01-01029.

\section{Список литературы}

[1] Дж. Майер, М. Гепперт-Майер. Статистическая механика. М.: Мир, 1980.

[2] Д. Рюәль. Статистическая механика. Строгие результаты. М.: Мир, 1971.

[3] Ф. Харари. Теория графов. М.: Мир, 1973.

[4] И. И. Иванчик. Метод ковариантного суммирования диаграмм в классической статистике. Дисс. докт. ф.-м.н. М.: ФИАН СССР, 1987; I. I. Ivanchik. Generalized Mayer Series in Classical Statistical Mechanics. New York: Nova Science Publishers, 1993.

[5] И. И. Иванчик. ТМФ. 1996. Т. 108. № 1. С. 135.

[6] Г. И. Калмыков. ТМФ. 1999. Т. 119. № 3. С. 475.

[7] O. Penrose. J. Math. Phys. 1963. V. 4. № 12. P. 1488. 
[8] Дж. Уленбек, Дж. Форд. Лекции по статистической механике. М.: Мир, 1965.

[9] Д. Н. Зубарев. ДАН СССР. 1958. Т. 118. № 5. С. 903.

[10] М. Дюно, Б. Суйар. Кластерные свойства решетчатых и непрерывных систем. В сб.: Гиббсовские состояния в статистической физике. Ред. Р. А. Минлос. М.: Мир, 1978. С. 89.

[11] М. Дюно, Б. Суйар, Д. Яголнитчер. Убывание корреляций в системах с бесконечным радиусом взаимодействия. В сб.: Гиббсовские состояния в статистической физике. Ред. Р. А. Минлос. М.: Мир, 1978. С. 107.

[12] Э. А. Аринштейн. ДАН СССР. 1957. Т. 112. № 4. С. 615.

[13] Э. А. Аринштейн. ДАН СССР. 1957. Т. 114. № 6. С. 1189.

[14] T. Morita. Progr. Theor. Phys. 1958. V. 20. № 6. P. 920.

[15] J. M. J. Van Leeuwen, J. Groeneveld, J. De Boer. Physica. 1959. V. 25. № 9. P. 742.

[16] T. Morita. Progr. Theor. Phys. 1960. V. 23. № 1. P. 175.

[17] T. Morita. Progr. Theor. Phys. 1960. V. 23. № 5. P. 829.

[18] T. Morita, K. Hiroike. Progr. Theor. Phys. 1960. V. 23. № 6. P. 1003.

[19] Э. А. Аринитейн, Б. Г. Абросимов. ЖКурн. структурной химии. 1968. Т. 9. №6. С. 1064.

[20] Г. А. Мартынов. ДАН СССР. 1974. Т. 218. № 4. С. 814.

[21] Г. А. Мартынов. ТМФ. 1975. Т. 22. № 1. С. 85.

[22] A. Н. Васильев. Функциональные методы в квантовой теории поля и статистике. Л.: Изд-во ЛГУ, 1976.

[23] И. И. Иванчик. Тр. ФИАН. 1980. Т. 124. С. 14.

[24] G. A. Martynov. Mol. Phys. 1981. V. 42. № 2. P. 329.

[25] И. И. Иванчик. Тр. ФИАН. 1984. Т. 144. С. 152.

[26] И. И. Иванчик. ДАН СССР. 1987. Т. 296. № 2. С. 341.

[27] И. И. Иванчик. ДАН СССР. 1988. Т. 300. № 3. С. 596.

[28] Г. И. Калмыков. ТМФ. 1990. Т. 84. № 2. С. 279.

[29] Г. И. Калмыков. Метод древесных сумм и его приложение к решению математических проблем классической статистической механики. Дисс. докт. ф.-м.н. М.: ВЦ РАН, 1998.

[30] J. Groeneveld. Phys. Lett. 1962. V. 3. № 1. P. 50.

[31] И. И. Иваниик. О бесповторном перечислении связных помеченных графов. В сб.: Комбинаторный анализ. Вып. 4. Ред. К. А. Рыбников. М.: Изд-во МГУ, 1976. С. 78.

[32] В. А. Мальшев, Р. А. Минлос. Гиббсовские случайные поля. М.: Наука, 1985.

[33] И. И. Иванчик. Тр. ФИАН. 1979. Т. 106. С. 3.

[34] A. A. Сапоженко. О числе связных подмножеств с заданной мощностью границы в двудольных графах. В сб.: Методы дискретного анализа в решении комбинаторных задач. Вып. 45. Ред. Ю. Л. Васильев. Новосибирск: Институт математики СО РАН, 1987. С. 42.

[35] Г. И. Калмыков. ТМФ. 1993. Т. 97. № 3. С. 452.

[36] Г. И. Калмыков. ТМФ. 1994. Т. 101. № 1. С. 94.

[37] Г. И. Калмыков. ТМФ. 1992. Т. 92. № 1. С. 139.

[38] Г. И. Калмыков. ТМФ. 1994. Т. 100. № 1. С. 44.

[39] Г. И. Калмыков. ТМФ. 1998. Т. 116. № 3. С. 417.

[40] Г. И. Калмыков. Каркасная классификация графов. В сб.: Труды IV Международной конференции "Дискретные модели в теории управляющих систем" (Красновидово, 19-25 июня 2000 г.). Ред. В. Б. Алексеев, В. А. Захаров. М.: МАКС Пресс, 2000. С. 34.

[41] Г. И. Калмыков. Каркасная классификация помеченных блоков. В сб.: Материалы VII международного семинара "Дискретная математика и ее приложения" (29 января - 2 февраля 2001 г.). Ч. ІІ. Ред. О. Б. Лупанов. М.: Изд-во Центра прикладных исследований при механико-математическом факультете МГУ, 2001. С. 221.

Поступила в редакцию 22.I.2001 г., после доработки 25.VII.2001 г. 